\title{
Curanderas a la sombra de la Huaca de la Luna
}

Guérisseuses à l'ombre de la Huaca de la Luna

Female Healers in the Shadow of the Huaca de la Luna

Bonnie Glass-Coffin, Douglas Sharon y Santiago Uceda

\section{(2) OpenEdition}

Journals

Edición electrónica

URL: http://journals.openedition.org/bifea/5815

DOI: $10.4000 /$ bifea.5815

ISSN: 2076-5827

\section{Editor}

Institut Français d'Études Andines

\section{Edición impresa}

Fecha de publicación: 1 abril 2004

Paginación: 81-95

ISSN: 0303-7495

\section{Referencia electrónica}

Bonnie Glass-Coffin, Douglas Sharon y Santiago Uceda, «Curanderas a la sombra de la Huaca de la Luna », Bulletin de l'Institut français d'études andines [En línea], 33 (1) | 2004, Publicado el 08 abril 2004 consultado el 01 diciembre 2020. URL : http://journals.openedition.org/bifea/5815 ; DOI : https:// doi.org/10.4000/bifea.5815

\section{(c) (i) $\odot$}

Les contenus du Bulletin de l'Institut français d'études andines sont mis à disposition selon les termes de la licence Creative Commons Attribution - Pas d'Utilisation Commerciale - Pas de Modification 4.0 International. 
Bull. Inst. fr. études andines

2004, 33 (1): 81-95

\title{
CURANDERAS A LA SOMBRA DE LA HUACA DE LA LUNA
}

\author{
Bonnie GLASS-COFFIN*, Douglas SHARON**, Santiago UCEDA***
}

\section{Resumen}

En este trabajo se presenta información arqueológica sobre representaciones de mujeres curanderas en ceramios Moche halladas en el complejo arqueológico Huacas del Sol y de la Luna.

El oficio de mujeres curanderas es un tema poco estudiado en la antropología cultural andina, y la presencia de representaciones de estas en época Moche demuestra una continuidad cultural entre el pasado prehispánico y el presente.

Sugerimos que la información recopilada sobre la simbología de las mesas de estas mujeres curanderas en este trabajo es una excelente ayuda para los arqueólogos e historiadores de las culturas prehispánicas, cuando buscan comprender los significados de contextos arqueológicos o reconstruir el pensamiento o ideología de ellas.

Palabras claves: Etnoarqueología, curanderas, Moche, Huaca de la Luna.

\section{GUÉRISSEUSES À L'OMBRE DE LA HUACA DE LA LUNA}

\section{Résumé}

Dans ce travail nous présentons des informations archéologiques sur les représentations de femmes guérisseuses sur des poteries trouvées dans le complexe archéologique des Huacas del Sol et de la Luna.

Le métier des femmes guérisseuses est un sujet peu étudié dans l'anthropologie culturelle andine, et la présence de représentations de celles-ci à l'époque Moche démontre une continuité culturelle entre le passé préhispanique et le temps présent.

Nous suggérons dans ce travail que l'information recueillie sur la symbolique de "las mesas" de ces femmes guérisseuses est un excellent apport pour les archéologues et historiens des cultures préhispaniques, pour pouvoir comprendre les significations des contextes archélogiques, ou reconstruire la pensée ou l'idéologie de ces dernières.

Mots clés Ethnoarchéologie, guérisseuses, civilisation Moche, Huaca de la Luna.

*Department of Sociology, Social work and Anthropology Utah State University, Logan, Utah 84322-0730. E-mail: glasscob@cc.usu.edu

** Phoebe A. Hearts Museum of Anthropology, University of California, Berkeley. Kroeber Hall 103, 3712. Berkeley, California 94720, USA.E-mail: dsharon@uclink.berkeley.edu

*** Proyecto Huaca de la Luna, Museo de Arqueología, Jr. Junín 682 Trujillo, Perú. E-mail: uceda@ddm.com.pe 


\title{
FEMALE HEALERS IN THE SHADOW OF THE HUACA DE LA LUNA
}

\begin{abstract}
This paper presents archaeological information about female healers or curanderas that are represented on Moche ceramics discovered in the archaeological complex of the Huaca del Sol and the Huaca de la Luna. The work of female healers is a little-studied topic in Andean cultural anthropology and the presence of these curanderas in the Moche period demonstrates cultural continuity between the pre-Hispanic past and the present. In this paper, we suggest that the information gathered about the mesa symbolism of these contemporary female healers can help archaeologists and historians who study pre-Hispanic cultures when looking to understand the significance of archaeological contexts or when reconstructing the world-view and ideology of past cultures.
\end{abstract}

Key words: Ethnoarchaeology, curanderas, Moche, Huaca de la Luna.

En agosto de 2001, en las excavaciones del sector residencial al pie de la Huaca de la Luna, Santiago Uceda encontró dos piezas cerámicas escultóricas Moche III que por sus rasgos físicos y vestimenta, y en un caso su pelo, parecen ser mujeres. Una lleva un disco y la otra una túnica con capucha y tiene en una mano una rodaja de lo que pudiese ser el cactus psicoactivo San Pedro. Estas piezas fueron descubiertas debajo de la ocupación asociada con cerámica del estilo Moche IV. Se trata de dos entierros complejos que corresponden a personal administrativo. La pieza representando una mujer con túnica proviene de la tumba 7 , la cual presenta dos momentos de entierro y cuatro individuos con ofrendas cerámicas. La pieza en mención estaba asociada con el individuo 3, que corresponde al segundo momento del entierro (Fig. 1); se trata de un feto cuya edad se estimó en nueve meses lunares. La segunda pieza que corresponde a la mujer sosteniendo al disco proviene de la tumba 10 que también tiene dos momentos, el primero (superior) es un entierro simple con abundantes restos de huesos humanos disturbados, pero ningún entierro completo. De este contexto proviene la pieza (Fig. 2). Por debajo de este entierro se halló una tumba de cámara poseyendo nichos en sus paredes este y oeste en un número de 7 en cada lado. En el lado oeste (hacia el mar) se encontró ceramios representando a las divinidades y en los nichos del lado este (hacia el continente) animales y seres humanos (Fig. 3).

La comparación de este contexto arqueológico con estudios etnoarqueológicos e iconográficos anteriores indican que las dos personas representadas pudiesen ser "curanderas" con posibles relaciones con la Mujer Mítica o Sacerdotisa de la Escena de Sacrificio (Sharon \& Donnan, 1974; Donnan, 1976; Donnan \& Castillo, 1992; Makowski, 1994; Sharon, 2000). A continuación presentaremos nuestras razones de pensar que las piezas representan curanderas y las implicaciones de esta tesis.

A nuestro criterio lo más llamativo de los datos arqueológicos son las asociaciones contextuales de las figuras. La que tiene la rodaja en la mano estaba al lado de huesos de una pierna humana y una niña que murió durante el parto. En el arte mochica hay bastante representaciones de mujeres encapuchadas sirviendo como parteras (Fig. 4). También hay ceramios donde, según Chávez (2000: 167, 171, 173) el niño nacido está envuelto al lado de la partera que lleva una rodaja de San Pedro en la mano (Fig. 5). 


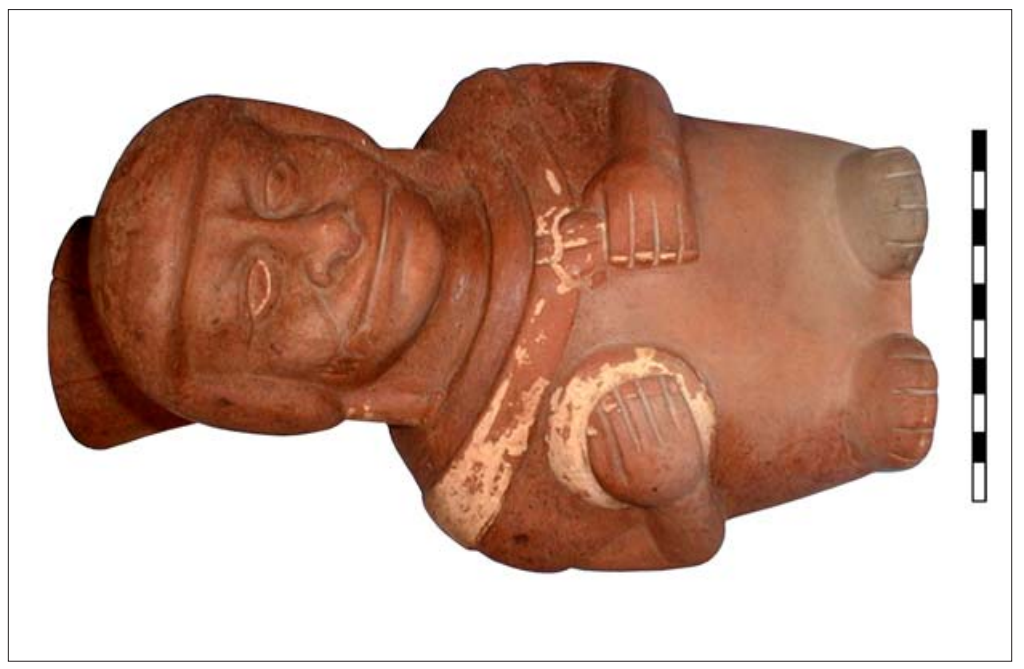

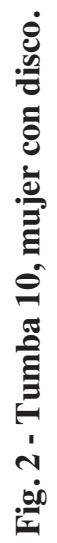

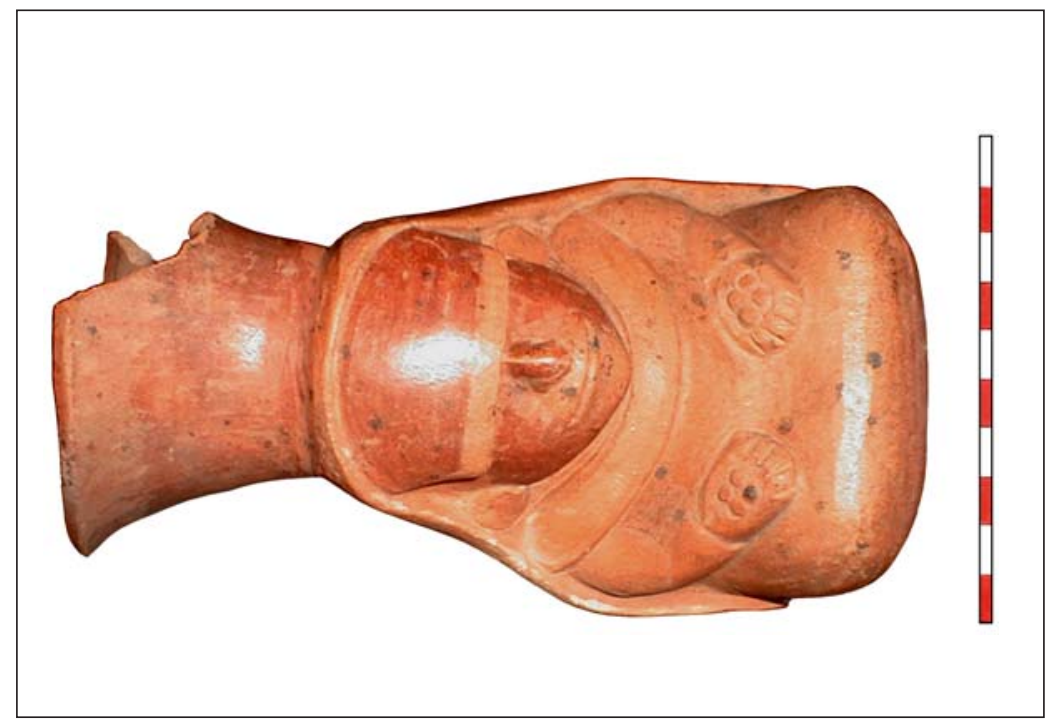

苞 


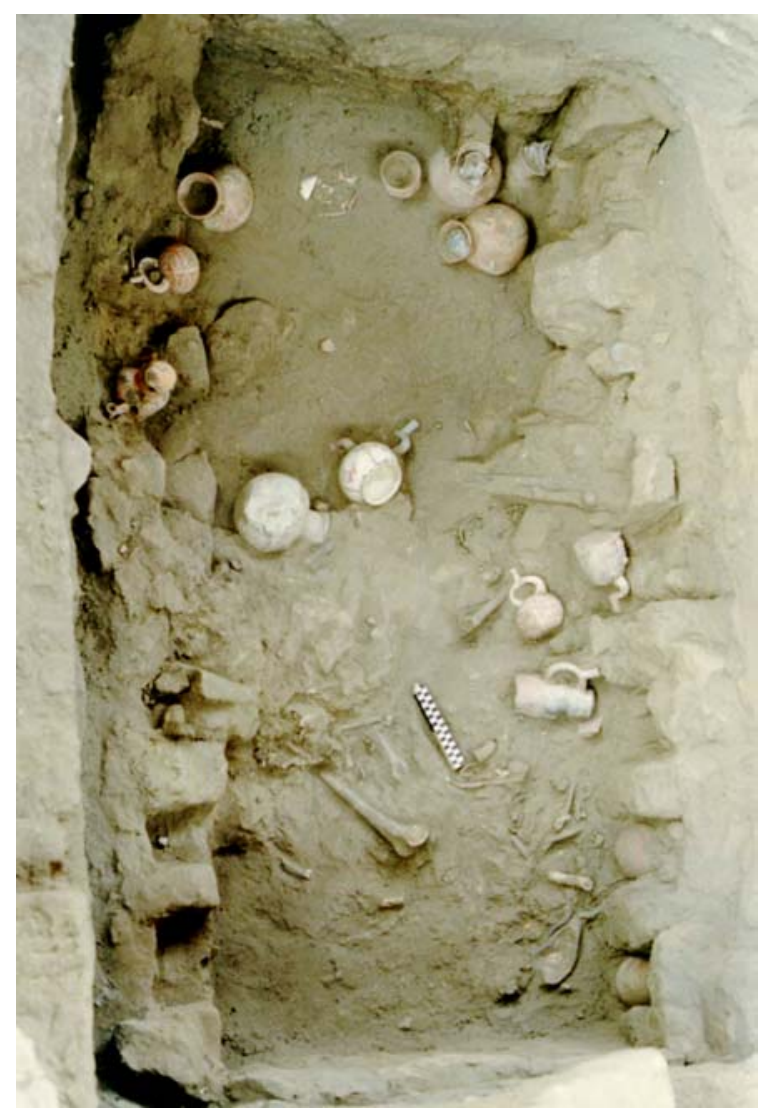

Fig. 3 - Tumba de cámara, ceramios de nichos en filas paralelas (este y oeste).

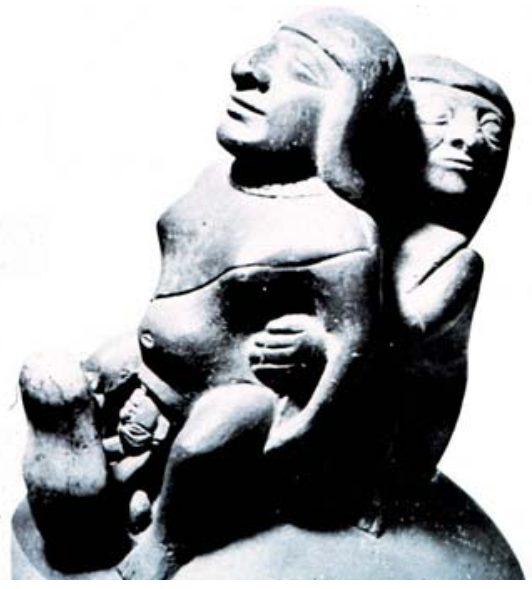

Fig. 4 - Mujer encapuchada como partera (Foto: Fernando Cabieses. Museo Larco Herrera, Lima). 


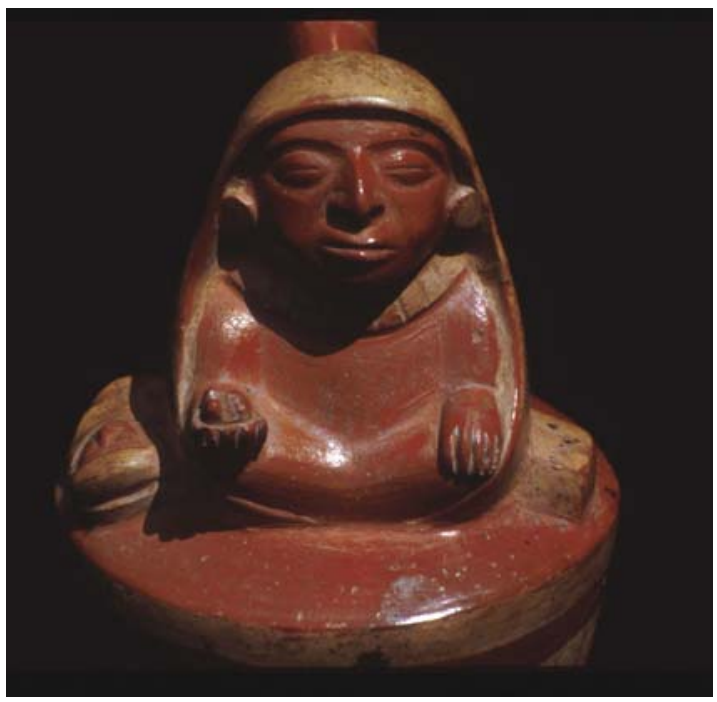

Fig. 5 - Mujer encapuchada con niño, caja y San Pedro en la mano (Foto: Douglas Sharon. Museo Larco Herrera, Lima).

Además, Bourget (2001: 102-105) sostiene que en algunos casos estas mujeres encapuchadas - especialmente las viejas arrugadas - están llevando niños para ser sacrificados a los ancestros.

En cuanto a la figura llevando un disco, ella tiene el cabello descubierto y escarificaciones en su cara. Además, lleva un bulto sobre sus espaldas. Está al lado de una cerámica de un prisionero. El disco que lleva es un elemento central en la Escena de Sacrifico junto con una copa de sangre (Fig. 6). En otras escenas de la iconografía mochica, una mujer con cabello descubierto se encuentra dentro de una audiencia adonde, en un caso, corren prisioneros desnudos (Fig. 7a); en el otro caso un prisionero está sentado fuera de la audiencia (Fig. 7b). Además de tener las manos libres, algunos prisioneros se llevan en literas que posiblemente indica cierto grado de participación libre en el acto de sacrificio (despeñamiento) o, por lo menos, una aceptación de la ideología religiosa que provee la justificación del rito. En la escena de la figura 7a los prisioneros corren atrás de la mujer con cabello descubierto en una audiencia ubicada debajo de una más grande ocupada por un señor que lleva una copa en la mano, y atendido por otro que lleva un disco. En la escena $7 b$ la audiencia de la mujer con cabello descubierto se encuentra entre dos otras donde quizás se lleva a cabo la presentación de copa y disco en distintas dimensiones (Makowski, 1994: 74) o por autoridades civiles y religiosas en este mundo.

Parece que la mujer con cabello descubierto es una especie de "consejera" o psychopompós para los que van a morir. Quizás la mujer con bulto y disco de la zona residencial de la Huaca de la Luna lleva el disco antes de la entrega a los sacerdotes o jefes. Es decir, pudiese ser la persona que facilita el sacrificio y aconseja a los prisioneros (representados por la cerámica del prisionero a su lado). El hecho de que ella se encuentra cerca de la mujer encapuchada asociada al parto parece indicar una relación 

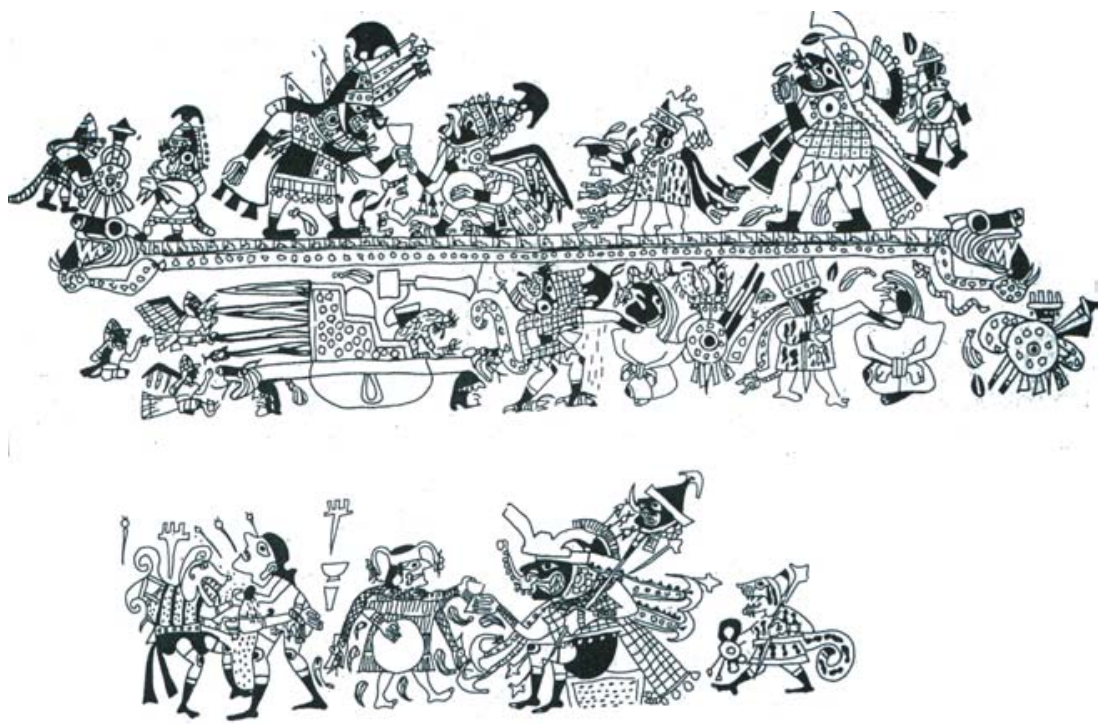

Fig. 6 - Discos en escenas de sacrificio (Donnan, 1976: figs. 104a y 106).

a)
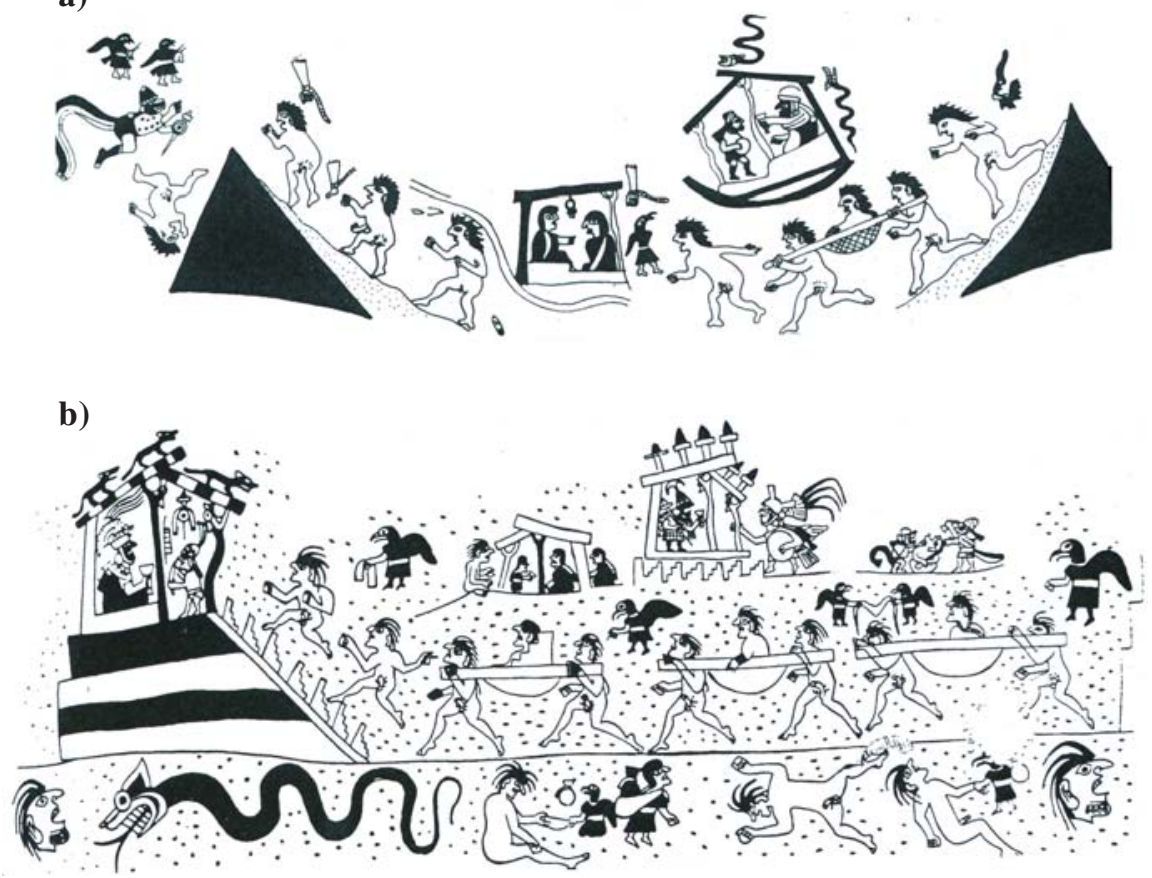

Fig. 7 - Mujer con cabello descubierto en audiencia. a: Prisioneros desnudos corriendo por los cerros con otra audiencia arriba (Bourget, 1995: fig. 18); b: Prisionero desnudo sentado afuera con dos audiencias a cada lado (Donnan, 1976: fig. 15). 
funcional entre las dos mujeres, es decir relacionadas con la muerte a la entrada y a la salida de este mundo.

Bourget (1995) ha propuesto que en los ritos mochicas las mujeres se asocian con la preparación del cuerpo, de ofrendas funerarias y de las víctimas. Agrega que "llevando niños sería una extensión de estas acciones" y que "los ritos funerarios y de sacrificio probablemente son cercanamente relacionados" (Bourget, 2001: 201).

En su perspicaz análisis de oficiantes femeninas en la iconografía mochica Makowski (1994: 57-58, 69-72, 81-83) distingue entre tres rasgos diagnósticos:

- cabello descubierto,

- cabello cubierto con un "pañuelo/turbante" y

-cabello cubierto con un "velo largo" que corresponden a la Representante de la Mujer Mítica, la Sacerdotisa y la Curandera, respectivamente.

Concluye que las mujeres con un velo largo "intervienen en el momento del pasaje, particularmente en el nacimiento y en la muerte, son probablemente ellas quienes descuartizan a los prisioneros sacrificados. [Nótese la pierna al lado de la mujer encapuchada de la tumba.] Una fuente iconográfica tardía de Lambayeque... las asocia con el uso de San Pedro..." (Makowski, 1994: 82). Las oficiantes de cabello descubierto "presiden ritos representando a la Mujer Mítica: bailan [con sonajas dobles], tejen [mantas ceremoniales], son fertilizadas en el transcurso de la unión carnal con el Mellizo Terrestre, se pelean con este dios, siendo representadas en el rol de madre, con un pequeño niño cargado a la espalda..." (Makowski, 1994: 82). Además aparecen en una de las versiones del Tema de Entierro y ordenan granos de maíz por colores en una manta (Makowski, 1994: 71-72) (Fig. 8). Makowski (1994: 82) sugiere que se trataba de una institución del tipo "acllas". Agregamos que posiblemente las mujeres con velo largo fueron similares a las "mamacunas".

En cuanto a la imagen de la mujer ordenando granos de maíz sobre una manta, Alva (2000: 29) la interpreta como "una prueba adivinatoria" donde la mujer con cabello descubierto cumple un rol de adivina. Para él existe una "asombrosa continuidad" entre el curanderismo actual del Norte del Perú y los conceptos y ritos shamánicos representados en la iconografía mochica tales como el parto, la adivinación, el diagnóstico de enfermedad, el uso de San Pedro y la transformación. Podemos agregar el rol de psychopompós. Según Alva (2000: 31-32) durante el ritual de curanderismo contemporáneo:

"El espíritu de los llamados "gentiles", es decir los ancestros prehispánicos son invocados junto a las tutelares montañas sagradas de cada región. Recordemos que en la cerámica Mochica es frecuente la representación del tema de sacrificio y ofrendas a las montañas, lugares de peregrinación, de donde viene el agua que fertiliza el desierto y donde moran las divinidades."

La manta con granos de maíz y el bulto de la mujer con pelo descubierto en la excavación - además de la presencia de cristales de roca - parecen ser similares a los envoltorios de "artes" del curandero de hoy:

"La llamada "mesa" de cada curandero actual está compuesta por una compleja y variada gama de objetos, artefactos y materiales de valor simbólico que son cuidadosamente dispuestos y manejados en el ceremonial, entre los cuales no pueden faltar ceramios, piedras (especialmente cristales), conchas spondylus o piezas metálicas, procedentes de los cementerios prehispánicos que proporcionan 


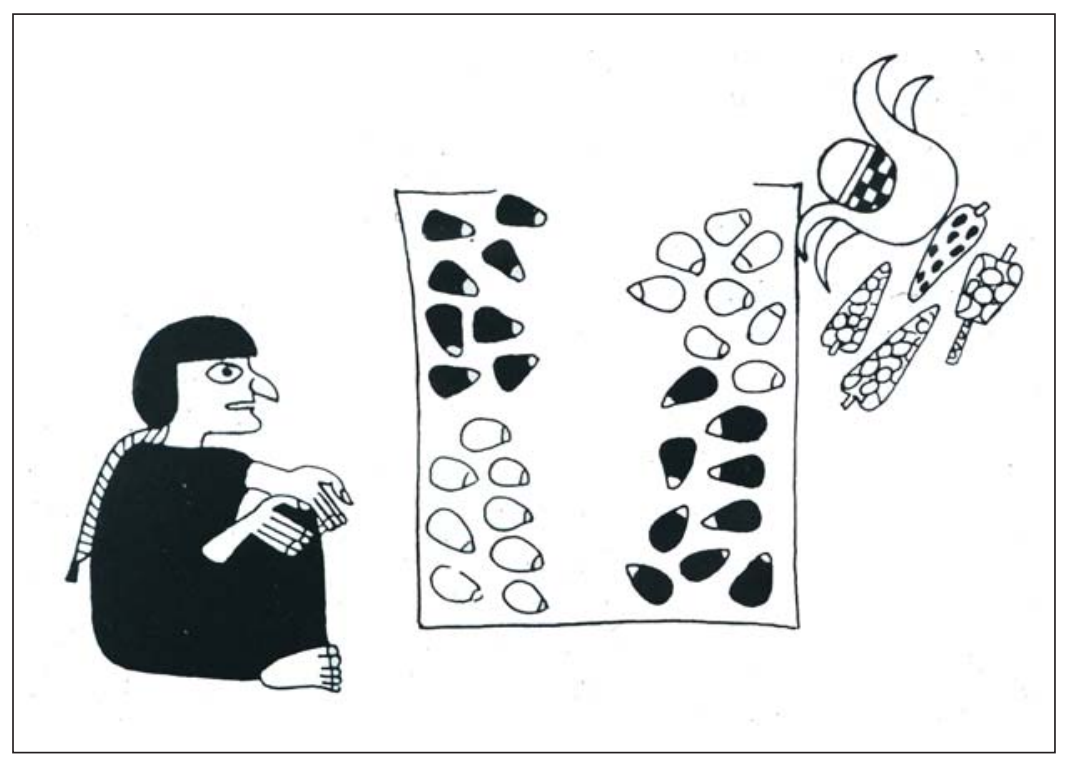

Fig. 8 - Mujer con cabello descubierto ordenando granos de maiz en una manta (Alva, 2000: dibujo 1).

poder a la "mesa" y al oficiante. Se conocen hallazgos de "mesas" asociadas a probables tumbas de curanderos prehispánicos altamente apreciada por los actuales. La disposición, la parafernalia ritual está regida por los conceptos de la cosmovisión Mochica (y andina), basada en el dualismo, referente a las dos fuerzas opuestas pero complementarias que rigen el universo, reflejadas en las oposiciones solluna, día-noche, negativo-positivo, macho-hembra, etc..." (Alva, 2000).

La cosmovisión dualística se manifiesta hoy en la división de la mesa norteña en dos campos: curandero y ganadero (Joralemon \& Sharon, 1994). Pensamos que también se manifiesta en las dos filas paralelas de cerámica encontradas debajo de las curanderas en la excavación del conjunto de la zona residencial al lado de la Huaca de la Luna. Forman dos líneas yuxtapuestas que denominamos "eje natural" y "eje sobrenatural" porque en una se encuentra el Dios de las Montañas representado en tres ceramios con un ave, un venado y un animal marino, es decir, en las tres pachas del cosmos andino, mientras que en el otro eje se encuentra escenas de la naturaleza y mujeres. También los opuestos complementarios se expresan en los dualismos de masculino-femenino y arriba (cerro)-abajo (mar) porque los dos ejes corren paralelos a la costa con uno al lado de los Andes y el otro al lado del mar.

Vale mencionar que la manta de la cerámica mujer con cabello descubierto de la Tumba 10 pudiese haber servido para cargar jarras de sangre como parece ser el caso en el sector bajo/central de la figura $7 \mathrm{~b}$.

Por último, queda el asunto de los papeles sociopolíticos que jugaban los personajes representados en los ceramios de este conjunto residencial al pie de la Huaca de la Luna. Pensamos que la transición de un entorno mágico-religioso (Moche III) a uno más administrativo (Moche IV) refleja el cambio de una sociedad tipo-señorío a un 
estado antiguo similar a lo que Alva (2000: 49) describe para Sipán, donde un examen detenido de atributos simbólicos, emblemas, ornamentos y atuendos rituales indica que:

"poseen reminiscencias formales de los conceptos y rituales shamánicos, indicando así la progresiva evolución del antiguo shamán como remoto líder espiritual hacia sacerdote-guerrero y finalmente a señores o gobernantes de efectivo mando político, ideológicamente sustentados por la casta sacerdotal".

También sostiene que la clase sacerdotal "convivía armónicamente en una jerarquía de roles con los líderes espirituales o shamanes de las comunidades menores..." (Alva, 2000: 33). Sugerimos que con el paso del tiempo las curanderas a la sombra de la Huaca de la Luna fueron suplantadas por la Sacerdotisa y su viaje mitológico y funerario por el "mar de noche" a las islas costeras con prisioneros y jarras de sangre, un tema dominante en Moche V.

Con los datos arqueológicos e iconográficos como trasfondo, ahora vamos a aplicar la analogía etnográfica para ver si podemos confirmar nuestra interpretación curanderil. Como se verá en adelante, hay una continuidad cultural muy arraigada desde el tiempo de los mochicas hasta la actualidad. Al hacer esto, partimos de la idea de Narváez (2001: 11) en su reciente y muy valioso aporte cuando afirma que el pasado "vive en el presente". En ese libro, Narváez presenta una colección de cuentos que son parte de la tradición oral lambayecana para ilustrar los cambios y la continuidad entre el registro arqueológico (ilustrado en los ceramios mochicas) y el presente. Como él afirma, "la tradición oral actualmente constituye una de las fuentes de mayor importancia para el estudio de la continuidad cultural" entre el pasado y el presente. Y esto sobre todo cuando se trata del "sincretismo religioso en el campesinado... [que] mantiene[n] elementos prehispánicos que han sido alterados, en mayor o menor grado, por la cultura occidental y cristiana; expresándose mediante un especial sincretismo" (Narváez, 2001: 27).

En el caso nuestro, después de encontrar los dos ceramios escultóricos, se pudo conversar con una curandera contemporánea llamada Ysabel que vive en la ciudad de Chiclayo, sobre el significado de estas cerámicas según su criterio. Las conversaciones fueron muy interesantes por varias razones. Primero, ella nos manifestó que había recibido un arte nuevo de un paciente unos meses atrás que tiene forma muy parecido al "disco" que se encuentra representado en la cerámica descrita anteriormente. Ella nos mostró el arte, que era una piedra redonda de color negro y que tenía un diámetro de aproximadamente 15 centímetros. Según ella, un paciente lo había encontrado en una tumba en la sierra de Huamachuco. En la figura 9 se ve la curandera con esa piedra en la mano derecha mientras alza una piedra cristal que también forma parte de su mesa en la mano izquierda.

Cuando recibe un arte nuevo, Ysabel acostumbra tomar el cactus San Pedro y trabajar con la mesa para entrar en trance y así poder ver el origen del arte. Es a través de ese diagnóstico que ella ve si podrá incorporar el arte nuevo como parte de sus herramientas de trabajo. También ve el nombre que debe darle y la función que tendrá cuando lo recibe como parte de su mesa.

Ysabel tiene muchas artes que ha incorporado a su mesa desde 1979, cuando empezó a trabajar como curandera. Entre ellas tiene artefactos "naturales" como son las conchas del mar, los cristales, piedras y varas de madera. También tiene artefactos "culturales" como son las espadas de metal, las macanas y los ceramios precolombinos, 


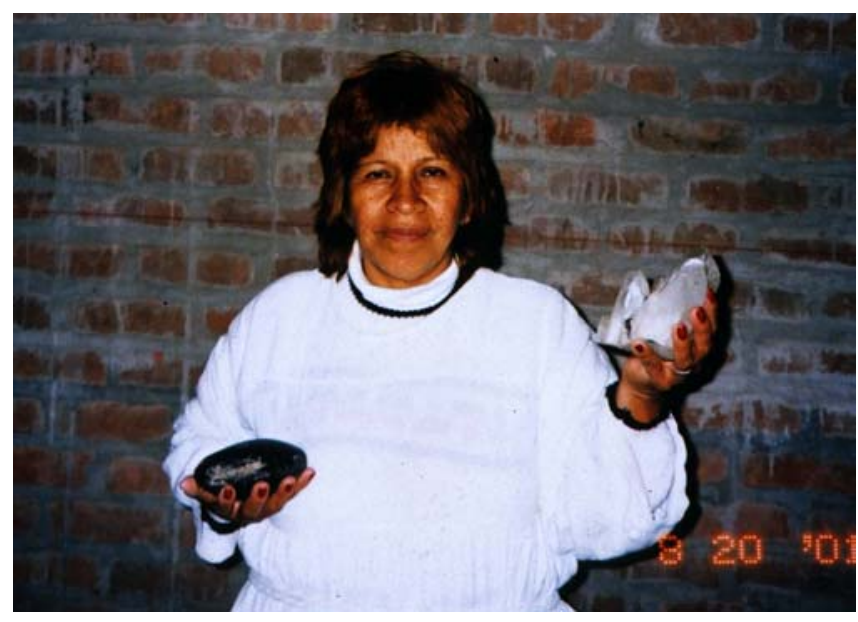

Fig. 9 - Ysabel muestra (en la mano derecha) el "disco" que ella llama "el pan" (Foto: Bonnie Glass-Coffin).

tanto como los crucifijos, las estatuas y los cuadros de santos. Aparte de estas artes su mesa contiene los líquidos que sopla para hacer ofrendas a los espíritus de los lagos, los cerros y las huacas que son conocidos como encantos. Y tiene la pócima del cactus San Pedro que sirve como el eje y como el fundamento de su habilidad de comulgar con estos encantos para el bien de sus pacientes (véase la Fig. 10 para el conjunto de objetos en su mesa; nótese la piedra "disco" en la parte centro/izquierda de la mesa).

Según Ysabel, todas los artes de su mesa tienen espíritus que los animan. Estos espíritus ayudan a la curandera en su labor de curar a sus pacientes. Para que los espíritus la apoyen, tienen que ser "pagados" con el líquido de tabaco, de maíz blanco, de flores blancas o de fragancias, según le indiquen. Generalmente, los espíritus de las piedras "naturales" se manifiestan como una energía o como la fuerza vital que une el ser humano con la tierra y con el cosmos. A veces esa energía se ve como la luz de los astros. A veces se ve como el remolino o el viento en movimiento. Hacer pagos a estas piedras facilita la circulación de esa energía entre los mundos de arriba, de abajo/adentro y de los seres humanos. De esa manera, facilita el enlace entre la humanidad y esa fuerza vital que los cristianos llaman Dios.

A veces los espíritus de sus artes se manifiestan como animales o como personajes humanos. Esto se ve especialmente vigente cuando se trata del espíritu de un arte que fue poseído y usado por un curandero antes de llegar a sus manos. Lo que Ysabel vio cuando contempló la piedra de Huamachuco era que había pertenecido a una curandera antigua, del tiempo de los mochicas. Ella describió el espíritu de esa mujer curandera — dueña de la piedra - así:

Es una reina, alta [como guerrera que maneja una tribu]... con pelo largo, con [dos] trenza[s]...[y] con cara... ni tan morena, ni tan blanca...

[Tiene] vestimenta de puro traje típico... túnico... que lindo... con colores... pardo, rojito, granate [nítido] como en tiras y largo hasta abajo... 


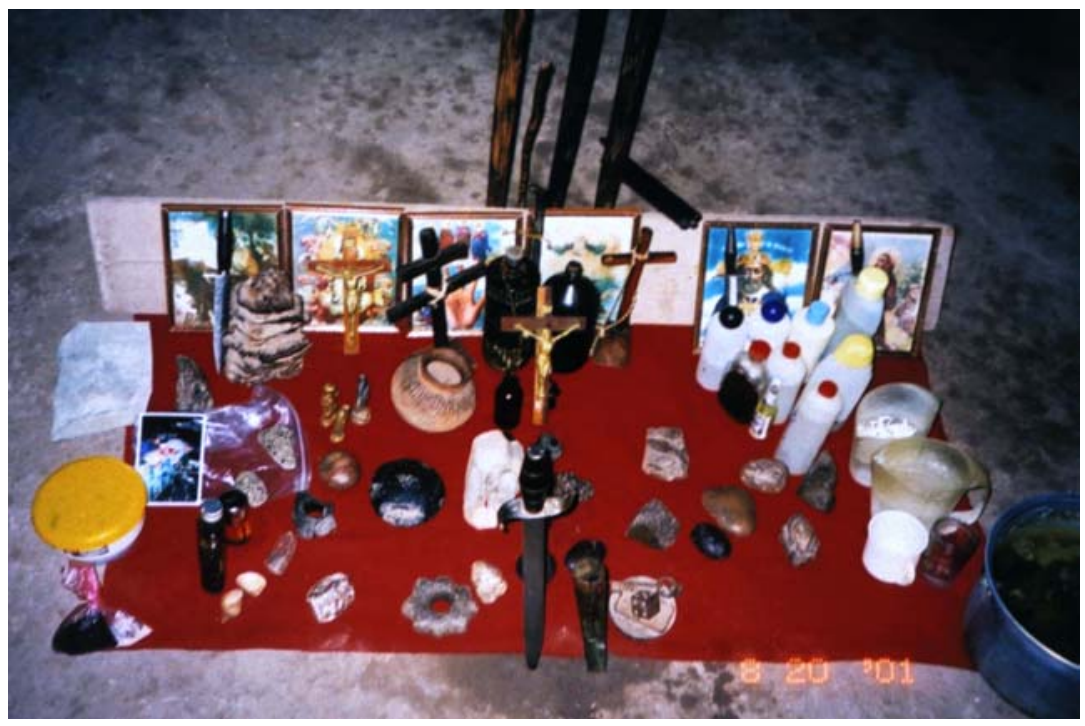

Fig. 10 - Mesa de Ysabel donde se ve el disco entre otros artes (Foto: Bonnie Glass-Coffin).

La toca de ella es como un manto negro pero [sobrepuesto] con colores... era en dos piezas porque la túnica era aparte y esto [el manto] era aparte...y [el manto] era su poder...

[C] uando ella va a poner su autoridad entonces se pone [el manto] y cuando va a quitar su autoridad se baja... por decir esto[el manto], es la corona [de la reina]...

[Lleva también]... collares de cerámica de muchos colores y sus orejeras acá son con cabezas de animales... como aretones... (Glass-Coffin, notas de campo/19 agosto, 2001).

Según Ysabel, ese arte fue utilizado por su dueña para hacer ofrendas a la naturaleza (la tierra) y a los astros (el cielo) con maíz blanco en polvo. Las ofrendas o "pagos" realizados con esta piedra fueron hechos como parte del ritual antecedente a un sacrificio humano. La curandera mochica jugaba el papel de "confesante" para preparar y purificar el prisionero destinado al sacrificio y actuaba como psychopompós que lo guiaba y lo reconfortaba en su viaje al más allá.

Cuando preguntamos a Ysabel sobre las correspondencias entre la manera en que la dueña utilizó esa piedra y la manera en que ella la utiliza ahora, nos respondió que es "casi una continuidad" porque antes lo utilizaron para hacer pagos u ofrendas y así lo utilizan ahora. La diferencia, según Ysabel, es que la curandera mochica utilizaba la piedra para hacer pagos antes de un sacrificio humano, mientras ella la utiliza para "el bien de la gente". Según ella, la curandera mochica debería de usar un lado de la piedra (que es como la parte "negativa" de aquella) para conseguir que los dioses reciban y hagan uso de un sacrificio humano. Esa piedra fue relacionada, por lo tanto con "la vida humana pero en sacrificio" o, en otras palabras, con la muerte humana. De forma contraria, 
Ysabel utiliza el otro lado de la piedra (que es como la parte "positiva" de aquella) para conseguir que los encantos respalden y fortalezcan las vidas de sus pacientes. Cuando le preguntamos qué nombre había puesto a la piedra como arte, nos dijo que lo había nombrado "el pan" porque está relacionada con buenas cosechas y con el pan que es necesario para sostener la vida humana. Insistía que la piedra tiene dos caras muy diferentes. La cara que ella utiliza tiene unas marcas que parecen raspaduras o arañazos en forma que ella describe como "espirales". Representan, para ella, "el camino de la vida humana". La otra cara es lisa y plana y ella concluye que fue esa cara que recibió los pagos de la curandera mochica. Concluyó su explicación de las correspondencias diciendo, "es muy importante esta pieza".

Son notables las semejanzas entre la cerámica de la mujer que lleva el disco encontrada por Santiago Uceda en la Huaca de la Luna y la manifestación del espíritu de la curandera mochica que Ysabel nos describió. Lo interesante es que Ysabel no vio la cerámica de la tumba antes de interpretarnos la visión que había tenido cuando vio la piedra que ella llama "el pan" en su mesa por primera vez. Ni pudo haber visto una foto de esa cerámica porque no nos fue posible llevar ni dibujo ni foto de las dos ceramios encontradas en la tumba unas cuantas días antes de nuestra visita. Pero, después de escucharla, intentamos describir los detalles de la cerámica para indagar sobre el significado que ella podría darnos. Le indicamos la manera en que la figura lleva el disco en la mano derecha tanto como la manera en que agarra un bulto que parece rebozo con la mano izquierda. Ysabel comentó lo siguiente:

"lo que sostiene acá [el disco] es de la tierra. Es de la naturaleza, y el manto que te estoy diciendo, ella lo utilizaba así, [agarrándolo como rebozo en la espalda cuando no está puesta en la cabeza]. Que era su poder y lo agarraba... el manto o el rebozo es el poder de algo..."

"Es que ellos utilizaban algún espíritu en la espalda o en la cintura, pero más era en la espalda... como decir algún poder, alguna arte... a veces así traían sus ofrendas también, porque no tenían bolsillos..." (Glass-Coffin, notas de campo/19 agosto, 2001).

Cuando le preguntamos acerca de los pagos que utilizaban los mochicas, Ysabel nos dijo que utilizaban maíz blanco, pero siempre en polvo porque no tenían líquidos en que macerarlo como suelen hacer los curanderos contemporáneos. Ponían los polvos encima de la piedra y los soplaron al aire de la misma manera que ella sopla el agua de maíz blanco sobre esa piedra en su mesa hoy día. Mientras los mochicas hacían los pagos a la tierra y a los astros, Ysabel hace los pagos "para los encantos de las huacas" de donde vienen las artes.

Enresumen,según la conversación que tuvimos con Ysabel,lacerámica representando a la mujer con disco encontrada por Santiago Uceda sería un retrato de la curandera mochica que tenía el oficio de preparar al prisionero para el sacrificio. Cuando le pregunté a Ysabel si la mujer con el disco tendría el cargo de hacer el sacrificio humano, me dijo, "por la mayor parte no... más preparaban para el sacrificio". El disco que lleva sería la manifestación del espíritu o de la fuerza vital de la tierra que recibiría pagos de maíz blanco para que reciba el sacrificio humano. El rebozo que se encuentra en la espalda de la cerámica sería un símbolo del poder de esa oficiante. Ella pondría ese manto encima de la cabeza "como corona de una reina" cuando le tocaba ejercer su poder.

No le conversamos a Ysabel acerca de las escarificaciones en la cara de la cerámica con disco, pero estos deberían de ser una manifestación del papel de esa mujer como 
shamana que sirve como mediador entre el mundo de los espíritus y el mundo de los humanos. Según Santiago Uceda (comunicación personal), las escarificaciones podrían ser interpretadas como una manifestación de la habilidad de la shamana de transformarse en un ser sobrenatural. En su papel de portero y de psychopompós hacía el mundo del "más allá", estas escarificaciones podrían servir como demostración de ese poder.

Ysabel también ha manifestado ese tipo de transformación durante sus rituales. En algunas de sus mesas cuando ha sido poseído por el espíritu del San Pedro su asistenta cuenta que hasta su cara se transforma y no se ve como la mujer joven que es sino que se ve como una viejita totalmente arrugada. En estas ocasiones, se transforma la curandera y habla con voz de viejita. Según su asistenta, es el espíritu del San Pedro - o más bien, el espíritu de Dios - que habla por la boca de Ysabel (Glass-Coffin, notas de campo, 1988-1989).

Después de conversar sobre esta cerámica, nos sentamos en una mesa con Ysabel para mostrarle unas fotos de otras ceramios que se han encontrado en unas tumbas de la cultura mochica. Entre ellas, le mostramos la curandera que tiene la cajita de piedritas al lado y una figura tendida adelante (Fig. 11; esta imagen ha sido publicada anteriormente en Glass-Coffin [1998] como foto $n^{\circ} 4$ entre las páginas 138-139). En primer lugar, le preguntamos a Ysabel si el personaje de la cerámica le parecía ser hombre o mujer. Nos dijo que era mujer, "porque todas las mujeres tienen este manto y su collar es asî".

Pero, según Ysabel, esta mujer no es la misma que la mujer del disco. Su manto es de una sola pieza y su oficio es de curar. Se nota que es la mujer curandera, según Ysabel porque tiene el paciente tendido y lo está frotando con las piedritas de la cajita que también sirven para hacer ofrendas. Según ella:

"Ellas [las curanderas mochicas] no curaban así frotando parado [como lo hago ahora] sino acostado... todo era acostado en la posición en que está. [Esto es a diferencia de los sacrificados porque] entonces para sacrificar... para prepararlo la curandera mochica así lo hacía parar, pero siempre inclinado, siempre de rodillas, pero para curar la mayor parte acostado..." (Glass-Coffin, notas de campo/19 agosto, 2001).

Le mostramos también, la famosa curandera Lambayeque que lleve un trozo de San Pedro en la mano (Fig. 12, publicada anteriormente como foto $n^{\circ} 2$ entre las páginas 138-139 de Glass-Coffin, 1998). Aunque ella no tenga un arte en su mesa curanderil que se parezca a esta cerámica, nos interesaba conocer su perspectiva sobre aquella por ser muy parecida a la otra cerámica encontrada por Santiago Uceda en la zona residencial de la Huaca de la Luna. Ella nos dijo que es el San Pedro que lleva en la mano y que el San Pedro fue el remedio principal para las curaciones en el tiempo de los mochicas como lo es ahora. Pero, a diferencia de la actualidad, las mujeres curanderas mochicas siempre utilizaban el cacto San Pedro de cuatro filos o cuatro "vientos". Ahora las mujeres utilizan el cacto de cinco o de seis filos - lo que corresponde más bien a los hombres curanderos - pero eso es porque es tan difícil encontrar los de cuatro filos.

Cuando le conversamos que esa mujer encapuchada con trozos en la mano fue encontrada en la tumba al lado de un bebé, nos dijo que no le sorprendía en absoluto. Nos hizo recordar de que uno de los papeles muy importantes de las mujeres curanderas es la de partera. Según ella, todo difunto - y más cuando se trata del entierro de un niñonecesitaría ser acompañado al mundo del más allá por ella, que sirve como mediador 


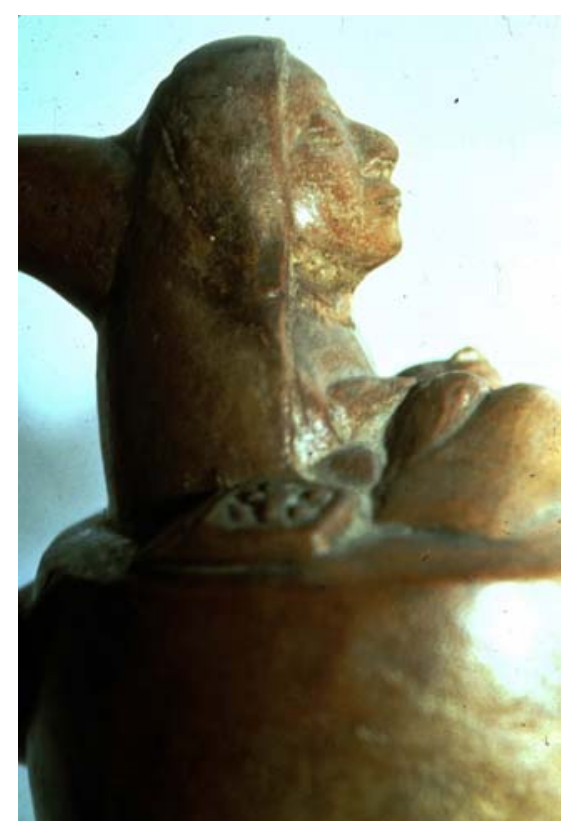

Fig. 11 - Cerámica de la cultura mochica donde una curandera utiliza piedras para curar a un paciente postrado

(Foto: Bonnie Glass-Coffin, Museo Nacional de Antropología, Lima, Catálogo \#C-54571).

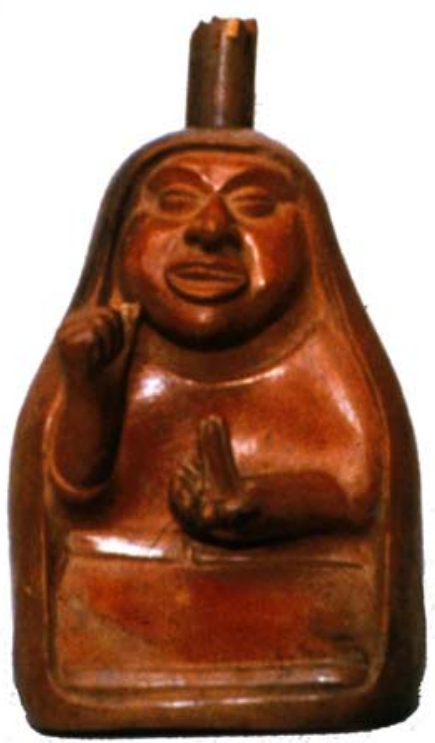

Fig. 12 - Cerámica de la cultura Lambayeque donde una curandera alza un pedazo de San Pedro en la mano

(Foto: Bonnie Glass-Coffin, Museo Larco Herrera, Lima). 
entre los dos mundos. En este caso, la mujer encapuchada con los trozos en la mano sería la persona que ayudaba en el parto y que podía reconfortar y guiar al infante en su viaje al otro mundo.

A pesar de no tener una escritura formal, nos parece que los mochicas tuvieron un sistema de comunicación visual y gráfico que expresaba sus ritos y creencias de una manera muy eficaz. Por medio de la etnoarqueología estamos comenzando a "leer" esta iconografía mochica. Es decir, una metodología de "texto y contexto" nos está revelando la cosmovisión de esta monarquía indígena. Sostenemos que este logro sea posible a raíz de la sorprendente continuidad cultural entre las curanderas mochicas de antaño y las curanderas mestizas contemporáneas de la costa norte del Perú. A pesar de los cambios sociopolíticos que han transcurrido desde la época prehispánica, ellas siguen facilitando el pasaje del umbral al otro mundo en los momentos "liminales" de su prójimo.

\section{Referencias citadas}

ALVA, W., 2000 - Curanderos, Shamanes y Sacerdotes el la Cultura Mochica. In: Shaman: La búsqueda... (L. Hurtado, ed.): 23-43; Cordóva: Imprenta de San Pablo S.L.

BOURGET, S., 1995 - Los Sacerdotes a la Sombra del Cerro Blanco y del Arco Bicéfalo. Revista del Museo de Arqueología, Antropología e Historia, 5: 81-125.

BOURGET, S., 2001 - Children and Ancestors: Ritual Practices at the Moche Site of Huaca de la Luna, North Coast of Peru. In: Ritual Sacrifice in Ancient Peru (E. Benson \& A. Cook, eds.): 93-118; Austin: University of Texas Press.

CABIESES, F., 1974 - Dioses y enfermedades: la medicina en el antiguo Perú, 357 p. +310 p., 2 tomos; Lima: Artgraf.

CHÁVEZ, F., 2000 - Sonadores, Terapeutas y carismáticas de los Andes del norte: un perfil Antropólogico. In: Shaman: La busqueda... (L. Hurtado ed.): 163-223; Cordova: Imprenta de San Pablo S.L.

DONNAN, C., 1976 - Moche Art and Iconography, 146 p.; Los Angeles: UCLA Latin American Center Publications.

DONNAN, C. \& CASTILLO, L., 1992 - Finding the Tomb of a Moche Priestess. Archaeology, 45(6): 38-41.

GLASS-COFFIN, B., 1989 - Apuntes de campo (inéditas).

GLASS-COFFIN, B., 1998 - The Gift of Life: Female Spirituality and Healing in Northern Peru; Albuquerque: University of New Mexico Press.

GLASS-COFFIN, B., 2001 - Apuntes de campo (inéditas).

JORALEMON, D. \& SHARON, D., 1994 - Sorcery and shamanism: curanderos and clients in northern Peru, 306p.; Salt lake City: Utah University Press

MAKOWSKI, K., 1994 - La figura del "oficiante" en la iconografía mochica: ¿shamán o sacerdote? In: En el Nombre del Señor: Shamanes, demonios y curanderos del norte del Perú (L. Millones \& M. Lemlij, eds.): 52-101; Lima: Australis S.A.

NARVÁEZ V., A., 2001 - Dioses Encantos y Gentiles: Introducción al Estudio de la Tradición Oral Lambayecana; Lambayeque: INC.

SHARON, D., 2000 - Shamanismo y el Cacto Sagrado; San Diego Museum papers 37.

SHARON,D.\& DONNAN,C., 1974 - Shamanism in Moche Iconography.In: Ethnoarchaeology (C. Donnan \& C. Clewlow, eds.): 51-77; Los Angeles: Institute of Archaeology, Monograph IV. 